\title{
Is one better than two?
}

In 1746, Dr. Pierre Fauchard wrote: "Those who are diligent on the conservation of their teeth and who wish to avoid being the victim of their error or their negligence ought to have them examined two or three times every year by an experienced dentist." This long-established assumption on dental recall has been challenged. According to Dr. Derek Richards, editor of Evidence-based dentistry, more evidence for the appropriate dental checkup visit interval is needed.

The recent study results of Dr. W. V. Giannobile published in the Journal of Dental Research set off a hot debate on the dental checkup interval issue. In this paper, he reported that getting one versus two dental checkups annually made no difference in tooth loss due to progressive periodontitis in low-risk patients, but made a significant difference in high-risk patients. Risk classification of the subjects was based on the history of smoking, diabetes, and the presence of the interleukin-1 genotype.

Some critics have claimed that these findings are not applicable to the general public because without a dental caries risk factor assessment and a direct check of patients' oral hygiene status, the optimal dental recall interval cannot be determined. However, we do have a basis for customizing dental recall times for patients: Clinical Guideline 19 from the National Collaborating Centre for Acute Care in the UK, which provides a 5 -step heuristic based on demographic, behavioral, and diagnostic information for determining an individual's proper dental recall interval.

It stands to reason that the issue of dental checkup intervals is truly worthy of debate given its public and private health impacts. However, we should not allow 
the debate should to be reduced to deciding on the optimal number of checkup intervals for the general public. Dr. Giannobile took pains to emphasize that his data that should not be interpreted as evidence for a certain dental recall interval, but rather that individualized treatment would be more effective than a standardized recall interval, both for those who need more frequent care and those who do not need checkups as often.

Therefore, our focus should be on finding useful measures for personalized dentistry. We welcome ongoing debate on the individualization of care based on additional clinical evidence.

\section{Tae-II Kim}

Editor-in-Chief

Department of Periodontology, Seoul National University School of Dentistry,

101 Daehak-ro, Jongno-gu, Seoul 110-749, Korea

E-mail: periopf@snu.ac.kr, Tel: +82-2-2072-2642, Fax: +82-2-744-1349 